Aus der Universitätsklinik und -Poliklinik für psychische und Nervenkrankheiten in Göttingen.

\title{
Die nervösen und psychischen Störungen
} bei Arteriosklerose.)

Von Geh. Med.-Rat Professor Dr. A. Cramer.

M.H.! Wie bei jeder Erkrankung. so ist auch bei der Arteriosklerose des Zentralnervensystems die Kenntnis der Frühsymptome von außerordentlich wichtiger Bedeutung, denn nur bei rechtzeitiger Erkenntnis der Krankheit ist ein therapeutischer Erfolg zu erwarten. Da es bei genau beobachteten Fällen nicht vorkommt, daß sich ohne prämonitorische nervöse Symptome auf dem Boden der Arteriosklerose eine psychische Störung entwickelt, will ich mich zunächst mit den nervösen Störungen der Arteriosklerose beschäftigen und erst später auf die psychischen Störungen eingehen.

Bei der Diagnose der Arteriosklerose spielt auch das Alter und das Vorleben eine Rolle. Ich muß das betonen, wenn wir auch im allgemeinen wenig Sicheres über die Aetiologie der Arteriosklerose wissen. ${ }^{2}$ ) Die meisten Fälle von Arteriosklerose des Zentralnervensystems treffen wir zwischen dem 45. und 65. Lebensjahre. Unter den Patienten sind viele, welche ein Leben voller Aufregungen, Anstrengungen und Strapazen hinter sich haben. Dabei können alkoholische Exzesse und Tafelfreuden, namentlich auch Vielesserei eine Rolle spielen. Auch übertriebene sportliche Betätigung kommt in Betracht. Allerdings können immer wieder Fälle vorkommen, wo alle diese Momente nicht besonders hervortreten und man auf eine angeborene geringere Widerstandsfähigkeit des Gefäßsystems hingewiesen wird, wenn man das Auftreten der Arteriosklerose verstehen will. Eine derartige endogene Disposition wird auch von den meisten Forschern, welche sich mit dieser Frage beschäftigt haben, betont. Häufig sind es sogenannte Kraftnaturen, die der Krankheit zum Opfer fallen.

Wenn wir unsere Fälle nach den Beruf sarten und Ständen betrachten, so stellen das Hauptkontingent Wirte, Schauspieler, Offiziere, Kommerzienräte, welche in vielen Aufsichtsräten sitzen; Beamte, welche viele repräsentative Verpflichtungen in Verbindung mit häufigen Aufregungen durch den Beruf haben, und nicht wenige unter unseren nervösen Unfallerkrankungen in der oben angegebenen Altersbreite.

Unter den nervösen Symptomen müssen wir unterscheiden zwischen Allgemein- und Lokalsymptomen.

Die Allgemeinsymptome gehen den deutlicher ausgeprägten nervösen Erscheinungen oft lange voraus. Die charakteristischsten sind die ominösen Trias Schwindel, Kopfschmerz und Abnahme des Gedächtnisses.

Das Symptom, das gewöhnlich alarmierend wirkt, wenigstens bei solchen Patienten, welche einigermaßen auf sich achten, ist der Schwindel, wenn auch der Kopfschmerz meist das Symptom ist, das allein den beiden anderen der Trias vorauseilt.

Der Kopfschmerz wird sehr verschieden geschildert, bald sind es anfallsweise auftretende bohrende Schmerzen an bestimmter Stelle, bald zeigen sich nur nach geistigen Anstrengungen früher nicht gewohnte "dumpfe“ Kopfschmerzen, bald fühlen die Kranken deutlich, wie ihnen nach einer Aufregung

1) Referat, erstattet auf dem XVI. Internationalen medizinischen KongreB in Budapest. - 2) Vgl. die Referate von Rom be rg und $M$ a r ch a nd auf dem Kongreb für innere Medizin in Wiesbaden im Jahre 1904 . 
ein Stich durch den Kopf fährt, der lange anhält, bald ist dauernd, bald schwankend ein diffuser oder lokalisierter Schmerz vorhanden, der die Arbeit erschwert und unter Umständen den Schlaf raubt. Manchmal bringen die Kranken auch das Auftreten des Kopfschmerzes von der Art der Nahrungsaufnahme und von einer gewissen Stuhlträgheit, einem gewissen Gefühl von Völle in den Bauchorganen oder von Blutandrang nach dem Kopfe in Abhängigkeit. Dieser Kopfschmerz kann, wenn therapeutisch nichts geschieht, wochen- und monatelang anhalten und dem Kranken außerordentlich lästig fallen.

Scharf wie durch einen Blitz wird das ganze Bild beleuchtet für den, der nur sehen will, wenn zu diesen hartnäckigen Kopfschmerzen, mögen sie nun auftreten, wie sie wollen, ein ausgesprochener $\mathrm{Schwindelanfall} \mathrm{sich} \mathrm{hinzugesellt.} \mathrm{Bei}$ diesem Schwindel handelt es sich um einen echten Drehschwindel, der Kranke muß sich festhalten, er hat Neigung $\mathrm{zu}$ fallen, oft wird er leichenblaß dabei, ja manchmal in besonders schweren Fällen stürzt der Patient auch zu Boden. Ja man kann manchmal zweifelhaft sein, ob man nicht die erste Attacke einer arteriosklerotischen Spätepilepsie vor sich hat. In allen diesen Fällen muß man immer damit rechnen, auch gelegentlich einen Neurastheniker oder einen endogen nervösen Hypochonder vor sich zu haben, der uns auch von Schwindel spricht, in Wirklichkeit aber nur einen der bekannten nervösen Schwächeanfälle hat, wie sie bei Nervösen häufig vorkommen und als Schwindel gedeutet werden. ${ }^{1}$ ) Ebenso muß man auch dieSchwindelanfälle ausschließen, welche durch Ohrerkrankungen bedingt sind. Man muß also genau examinieren, wenn man nicht vor Fehlschlüssen bewahrt bleiben will. Diese echten Schwindelanfälle zeigen sich in verschiedener Häufigkeit; im Anfang selten, alle paar Monate auftretend, können sie in besonders schweren Fällen mehrmals täglich sich zeigen. Auch sind sie meist von wechselnder Intensität. Weisen Kopfschmerz und Schwindel wirklich auf Arteriosklerose hin, so fehlt auch meist die Abnahme des Gedächtnisses nicht. Im Anfang hat die Gedächtnisschwäche wenig Charakteristisches. Die Kranken klagen lediglich darüber, daß ihnen manchmal ein Name nicht einfällt, daß sie sich auf ein Wort nicht besinnen können, und geben an, daß das häufiger als früher sei und zunehme. In ausgeprägteren Fällen kommt dazu, daß sie namentlich bei öffentlichem Auftreten, während sie früher gute Redner waren, den Faden der Rede verlieren und nicht weiter können. Ich habe Fälle gesehen, wo solche Erscheinungen jahrelang dem Ausbruch der übrigen Erscheinungen vorausgingen. Bei schwereren, fortgeschritteneren Fällen kann es dann zur Ausbildung des amnestischen Symptomenkomplexes kommen. (Verlust des Gedächtnisses für die jüngste Vergangenheit und Konfabulation.) Meist allerdings besteht lediglich die Erscheinung, daß die jüngsten Erlebnisse außerordentlich wenig und kurz haften bleiben. Es passiert deswegen den Patienten auf der Bahn und in den Hotels nicht selten, daß sie sich nicht mehr in ihr Abteil oder in ihr Zimmer zurückfinden können. Namentlich geraten sie leicht in Verlegenheit, wenn es im Verkehrsleben gilt, sich Nummern zu merken. Sie schreiben sich auch alles, was sie behalten wollen, auf und sind ohne diese Notizen nur schwer imstande, eine genauere Anamnese anzugeben. Meist kommt deshalb die Frau oder ein anderer Verwandter mit, um zu berichten.

Ein charakteristisches Allgemeinsymptom, das wir fast in allen ausgeprägteren Fällen mehr oder weniger deutlich ausgesprochen finden, ist schließlich noch die Rührseligkeit. Wie bei jeder organischen Gehirnerkrankung, häufig auch im Alter und seltener bei schwer endogen disponierten Fällen, so beobachten wir auch bei unseren Arteriosklerotikern, daß sie sehr leicht gerührt, ja zu Tränen gerührt werden. Sie ärgern sich oft über diese Eigenschaft, die sie früher nicht kannten, und kommen deswegen in unsere Sprechstunde, weil sie bei allen möglichen und unmöglichen Gelegenheiten schluchzen und in Tränen ausbrechen müssen. Es passiert ihnen das im Theater, während des öffentlichen Sprechens, in der Familie und im Dienst in außerordentlich störender Weise. Differentialdiagnostisch kann natürlich dieses Symptom allein ebenso-

1) In meinem Buche über Nervosităt habe ich 7 Varietäten von nervősem Schwindel beschrieben. wenig wie die anderen in Betracht kommen, es kann uns nur aufmerksam machen, genauer zuzusehen. Ausgeprägtes Frühsymptom ist die Rührseligkeit seltener. Als weitere Allgemeinsymptome, die aber nicht so konstant sind wie die bisher erwähnten Erscheinungen, kommen noch in Betracht: zunächst ein zeitweise auftretendes, mehr oder weniger ausgeprägtes Eingenommensein des Kopfes, das sich manchmal fast einer Benommenheit nähert. Ferner leichte Zustände von Depres sion und Schwarzseherei, die im früheren Leben sich nicht gezeigt haben, sehr selten sind mehr euphorische Zustände. Häufiger aber finden wir, daß sich die gesamte Individualität des Patienten insofern verändert, als er stumpfer und gleichgültiger wird. Sein Interesse läßt nach, ebenso seine geistige Beweglichkeit. In anderen Fällen sehen wir, daß sich eine stärkere Reizbarkeit einstellt, nicht nur gegen Licht- und Schallreize, sondern namentlich auch gegenüber den kleinen Ereignissen und Aergernissen des alltäglichen Lebens. In wieder anderen Fällen stellt sich ein übertriebener Hang zur Sparsamkeit ein, ohne daß etwa von Verarmungsideen gesprochen werden kann. In solchen Fällen haben, namentlich wenn gleichzeitig noch eine gesteigerte Reizbarkeit besteht, die Frau und die erwachsenen Kinder ganz besonders zu leiden.

Der Schlaf verhält sich in den einzelnen Fällen sehr verschieden. Manchmal geht eine quälende Schlaflosigkeit lange Zeit voraus, manchmal sind, wie wir später sehen werden, die Kranken förmlich schlafsüchtig. Häufig berichten uns auch die Patienten oder ihre Angehörigen, daß sich ihre Toleranz dem Alk ohol gegenüber völlig verändert habe, daß sie nicht einmal mehr ein Glas Wein vertragen können und bei solchen Gelegenheiten zum Genuß geistiger Getränke auffallen, was früher nie vorgekommen sei. In selteneren Fällen erfahren wir auch, daß sie immer egoistischer geworden seien. Daß sie nur noch an sich denken und für nichts mehr Interesse haben. Ist dies Symptom sehr ausgeprägt, so sehen wir, daß oft alles andere gegenüber diesem krassen Egoismus lange Zeit zurücktritt. 'Die Kranken sprechen nur von sich und sind nur interessiert, wenn von ihnen die Rede ist. Es ist ihnen kaum möglich, einer Unterhaltung zu folgen, welche sich nicht mit ihnen beschäftigt.

In selteneren Fällen können auch frühzeitig die ethischen Vorstellungen zurücktreten, die Patienten werden verlogen und wissen namentlich, wenn sexuelle Begehrlichkeiten auftreten, mit großem Geschick ihren sexuellen Abenteuern das Deckmäntelchen christlicher Liebe und Barmherzigkeit umzuhängen. Oft wissen nur die Angehörigen, was für ein laszives Leben der Patient im Geheimen führt. Nähert sich dieser Zustand mehr der arteriosklerotischen Psychose, dann fällt die letzte Kritik und Zurückhaltung, und es kommen in solchen Fällen die strafrechtlich zu verfolgenden Sittlichkeitsvergehen und Schamverletzungen. Alle diese Fälle, welche glücklicherweise, wie schon betont, selten sind, machen, solange ausgesprochene psychische Störungen noch nicht nachweisbar sind, yroße Schwierigkeiten in der forensischen Beurteilung. Wir sehen also, daß sehr gelegentlich Züge aus der später zur Entwicklung gelangenden Psychose als Frühsymptom und Allgemeinsymptom die nervösen Störungen der Arteriosklerose komplizieren können.

Auch sei hier nochmals ausdrücklich betont, daßjedes Allgemein- und Lokalsymptom gelegentlich Frühsymptom sein kann.

Unter den Lokalsymptomen kommen vor allen Dingen Erscheinungen in Betracht, welche als Ausfalls- und als Reizessymptome gedeutet werden müssen, kurz gesagt, Stigmata, welche auf eine Neuronerkrankung hinweisen.

Nicht selten habe ich hier als Frühsymptom eine auffallende Verlangsamung der Sprache gesehen. Ich meine nicht eine Erschwerung der Sprache, sondern lediglich einen langsameren Ablauf des Sprachvorganges. Während normaliter $z$ wischen 200 und 300 Silben in der Minute gesprochen werden, sinkt in diesen Fällen die Silbenzahl auf 100 und sogar auf 80 . Bei Patienten, welche häufig in der Oeffentlichkeit sprechen müssen, sind es gewöhnlich die Stenographen, denen die Verlangsamung im Sprechen, welche sie als Wohltat empfinden, auffällt. In mehreren Fällen habe ich dieses Sym- 
ptom mehrere Jahre dem Auftreten anderer Symptome vorausgehen sehen. Begreiflicherweise ist diese Erscheinung an und für sich, wenn nicht noch andere Symptome vorhanden sind, zunächst noch nicht von der langsamen Sprache des bedächtigeren Alters zu unterscheiden.

Häufig gesellt sich dazu noch, namentlich in den ausgesprocheneren Fällen, eine Erschwerung und ein Undeutlichwerden der Sprache. Ohne dab eine ausgesprochene artikulatorische Sprachstörung vorhanden ist, wird die Sprache verwaschen, es stellt sich ein leichtes Tremolieren ein, namentlich die letzten Worte und Silben eines Satzes werden undeutlich und zum Teil verschluckt. Diese Erscheinungen fallen um so mehr auf, wenn sich damit zugleich, wie das häufig ist, eine Verlangsamung der Sprache verbindet. Meist ist damit auch verbunden ein mehr oder weniger ausgeprägtes Mitflattern der Gesichtsmuskulatur, ganz ähnlich wie wir es bei der progressiven Paralyse, bei anderen organischen Gehirnerkrankungen und bei starker Erschöpfung sehen können.

Sehr wichtig sind auch die Veränderungen an den Pupillen. Es handelt sich um eine Trägheit der Pupillenreaktion, wie wir sie so ausgeprägt in der betreffenden Altersbreite sonst nicht $\mathrm{zu}$ sehen bekommen. Meist ist dabei nicht nur die Reaktion auf Lichteinfall, sondern auch die auf Akkommodation betroffen. Fälle, bei denen die Pupillen tadellos reagieren, sind sehr selten. Weniger häufig sehen wir verzogene oder nicht ganz runde Pupillen. Charakteristisch ist für diese Pupillenstörungen nicht nur das gleichzeitige $\mathrm{Be}$ stehen einer trägen Reaktion oder in seltneren Fällen sogar Starre auf Lichteinfall und Akkommodation, sondern vor allem auch, wie Weber ${ }^{1}$ ) mit Recht betont, das Schwanken in der Intensität der Erscheinungen. Die Reaktion der Pupillen kann nicht nur im Verlauf von Wochen und Monaten sich verschieden verhalten, sondern auch an einem und demselben Tage, zu verschiedenen Stunden ein ganz verschiedenes Verhalten zeigen.

Garnicht selten sieht man auch frühzeitig eine Differenz in der Fazialisinnervation. Allerdings muß man sich hier hüten, wenn man dem Symptom eine diagnostische Bedeutung zuweisen will, der Verwechslung einer angeborenen Fazialisparese zum Opfer zu fallen. Sehr ausgeprägt sind diese Paresen im Anfang meistens nicht, sondern nur angedeutet; erst im weiteren Verlauf der Krankheit, wenn stärkere zerebrale Insulte stattgefunden haben, markieren sie sich deutlicher.

Andere Gehirnnervenlähmungen sind selten. Gelegentlich kommen auch als Frühsymptom Augenmuskellähmungen vor, manchmal direkt in Gestalt einer Ophthalmoplegie, wie sie seinerzeit von Siemerling und Boedecker beschrieben worden ist. Am häufigsten beobachtet man nach meinen Erfahrungen flüchtige und allmählich konstanter werdende Abduzensparesen.

Die Reflexe sind meistens gesteigert. Was speziell das Kniephänomen betrifft, so kann unter Umständen, wenn auch selten, ein differentes Verhalten vorkommen, ja es kann auch fehlen. Die Befunde, welche A. Westphal in diesem Frühjahr auf der Versammlung des Deutschen Vereins für Psychiatrie demonstrierte, lassen dieses eigentümliche Verhalten versitehen. Denn, wenn die Arteriosklerose im Rückenmark herdförmige Erkrankungen hervorrufen kann, die in ihrer Verteilung durchaus denen der multiplen Sklerose ähnlich sind, wie die Untersuchungen von Westphal bestimmt erweisen, so kann es keinem Zweifel unterliegen, daß wir auch entsprechende Symptomenkomplexe klinisch auffinden müssen. Wiederholt sind an meiner Klinik, ohne ausgesprochene Halbseitenläsionen oder Monoplegien, isolierte, leichte Spasmen, ja sogar isoliertes Auftreten des Babinski beobachtet (W eber). Diese Erscheinungen, welche im Anfang nur flüchtig und intermittierend vorhanden waren und erst später konstant wurden, sind zunächst am besten unter dem Gesichtspunkt der von Westphal erhobenen Befunde zu verstehen.

Nicht selten klagen die Kranken auch über allerlei Parästhesien und Schmerzen, namentlich an den Extremitäten

1) We ber, Zur Klinik der arteriosklerotischen Seelenstorrung. Ziehens Monatsschrift, Bd. 23, Ergänzungsheft S. 175 . - D erselbe Arteriosklerotische Verstimmungszustände. Münchener medizinische Wochcnschrift 1909, No. 30 . S. A. ebenda auch Literatur. und auch an anderen Teilen des Körpers. Sie haben eigentümliche, fremdartige Gefühle an den Händen und Füßen, in den Armen und Beinen. Das Gefühl von Taub- und Pelzigsein, Ameisenlaufen, Spannungen, eigentümliche Kältegefühle und direkt stechende, bohrende Schmerzen werden angegeben. Eine Prädilektionsstelle für sehr unangenehme Schmerzen sind oft die Fersen, ohne daß irgend etwas nachzuweisen ist.

Alle diese Erscheinungen können ja auch auf rein nervöser Basis vorkommen, wir dürfen sie erst per exclusionem und wenn wir auch andere Symptome der Arteriosklerose nachweisen können, auf unsere in Rede stehende Krankheit beziehen. Dabei macht uns oft genug noch die Diagnoseentscheidung Schwierigkeiten, ob wir es ledigich mit einem peripherischen arteriosklerotischen Prozeß zu tun haben, wie z. B. bei dem intermittierenden Hinken, oder mit einem zentralen. Wir müssen es uns überhaupt zum strengen Gesetz machen, daß wir niemals die Diagnose Arteriosklerose stellen, ohne dab wir alle Hilfsmittel der Diagnostik, welche uns die innere $\mathrm{Me}$ dizin bietet, zu Hilfe nehmen. (Veränderungen am Herzen, Verbreiterung nach links, klappender Aortenton, unreine Töne, Blutdrucksteigerung, gespannter Puls, eventuell entsprechende Nierenveränderungen).

Oft genug wird die ganze Erscheinungsreihe, die ich bisher geschildert habe, übersehen, nicht genügend beachtet und in ihrer Bedeutung nicht gew ürdigt. Alsdann sind es die Erscheinungen eines Insultes, welche plötzlich den Patienten und seine Umgebung jüh aus ihrer Ruhe emporschrecken. Diese Insulte melden sich manchmal vorher an, indem wiederholt leichtere Spannungen und Parästhesien auftreten, indem die Kopfschmerzen und der Schwindel sich verstärken, indem die Patienten mehr benommen erscheinen, ängstlich sind oder über Schweratmigkeit und Herzbeklemmung klagen, sie können aber auch gänzlich unvermutet sich zeigen. Sie sind der klinische Ausdruck für die Herderkrankungen des Gehirns, auf welche schon frühzeitig Alzheimer und Binswanger in ihren klassischen Arbeiten über die Arteriosklerose hingewiesen haben.

Begreiflicherweise sind die klinischen Allgemeinerscheinungen verschieden, je nachdem wir Blutungen oder Erweichungen vor uns haben. Ferner sei betont, daß auch Embolien und Thrombosen gelegentlich eine Rolle spielen können, obschon diese letzteren Verhältnisse für unsere Betrachtungen mehr zurücktreten. Ich möchte aber bei dieser Gelegenheit erwähnen, daß ich kürzlich einen Fall von arteriosklerotischer Atrophie beobachtet habe, bei dem das Verschwinden ausgeprägter aphasischer Erscheinungen bei der Sektion (Brown) durch Kanalisierung von Thromben aufgeklärt wurde. Die Blutungen und Erweichungen machen je nach Größe und Sitz ganz verschiedene Erscheinungen, namentlich Lokalsymptome. Die Allgemeinsymptome sind selbstverständlich bei den Blutungen stürmischere als bei den Erweichungen. Da aber meist beide Prozesse vereinigt sind oder doch gleichzeitig in verschiedenen Teilen des Gehirns sich abspielen, erhalten wir häufig sehr gemischte Symptomenkomplexe. Daß plötzliche, starke apoplektische Insulte den Kranken ohne Vorboten überfallen, ist bei der arteriosklerotischen Atrophie selten. Meist handelt es sich zunächst um flüchtige, passagere Erscheinungen, leichte, rasch wieder verschwindende Mono- oder Hemiparesen, transitorische aphasische Erscheinungen, Hemianopsien und Sehstörungen, die rasch wieder verschwinden, auch flüchtiges Doppeltsehen für kurze Zeit und andere ähnliche Symptome. Wenn diese Erscheinungen auch so auftreten können, daß sie gleich konstant bleiben, so ist doch nach meinen Erfahrungen häufiger, daß sie bald kommen, bald gehen, um endlich, wenn nicht energisch therapeutisch eingegriffen wird, konstant zu werden. Wiederholt habe ich Fälle gesehen, wo sich monoplegische oder hemiplegische Erscheinungen mit ausgesprochenen sensorischen Reizerscheinungen verbanden: Hören von Musikstücken, Sehen von mehreren schwarzen Linien, die durch das Gesichtsfeld ziehen, intensive Kältegefühle an bestimmten Stellen des Körpers, namentlich im Gesicht und Nacken der einen Seite. Findet sich der arteriosklerotische Prozeß mehr in Medulla oblongata und Brücke lokalisiert, so können die Erscheinungen der progressiven Bulbärparalyse das Krank- 
heitsbild komplizieren. Bei noch tieferem Sitz kann auch der Brown-Séquardsche Typus auftreten. Auffällig ist, daß sich gelegentlich der arteriosklerotische Prozeß mehr auf einen Teil oder eine Hälfte des Gehirns beschränkt; in solchen Fällen sind von vornherein konstante Lokalsymptome vorhanden. ${ }^{1}$ )

Dieses ist mir namentlich in Fällen von arteriosklerotischer Spätepilepsie aufgefallen, wenn wir auch diesen Symptomenkomplex bei mehr diffuserer Verbreitung der Arteriosklerose zu sehen bekommen.

In einem Falle, bei dem es sich um eine ausgesprochene Spätepilepsie handelte, welche in ihren Anfällen durch ein einleitendes Stadium von Jacksonschem Typus ausgezeichnet war und be welchem anfangs lange Zeit Zustände völliger Luzidität mit solchen schwerer Verwirrung abwechselten, um schließlich einem Zustande dauernder schwerer Benommenheit Platz zu machen, fand ich den hinteren Teil des Stirnlappens, die beiden Zentralwindungen und den vordersten Teil des Scheitellappens der entsprechenden Seite durch Erweichungen verändert.

Bei dieser Gelegenheit möchte ich bemerken, daß man eine Spätepilepsie nur diagnostizieren darf, wenn ausgesprochene epileptische Anfälle mit Zungenbiß und den anderen klinischen Erscheinungen nachgewiesen sind, daß wir dagegen die Ohnmachtsanwandlungen, ja auch ein gelegentliches Umfallen, wie es bei der Arteriosklerose bisweilen vorkommt, noch nicht als Spätepilepsie auffassen dürfen. Auch die posthemiplegischen Bewegungsstörungen berechtigen nicht ohne weiteres zu dieser Diagnose.

Die Allgemeins y mpto me, welche die Herderscheinungen bei der Arteriosklerose begleiten, sind bekannt. Vermehrtes Auftreten von Schwindel und Kopfschmerzen, Ziehen und Reißen in den Extremitäten, wie Parästhesien an den verschiedensten Stellen des Körpers, Blutandrang nach dem Kopf, Brechneigung, Ohnmachten, Neigung zum Umfallen, Unfänigkeit sich aus dem Bett aufrichten zu können, wegen zu starken Schwindels, mehr oder weniger schwere Benommenheit, Incontinentia urinae et alvi und ähnliches.

Als Begleiterscheinungen kommt noch eine Reihe von anderen Erscheinungen in Betracht, welche weniger von zentralen Veränderungen in Abhängigkeit gebracht werden können. Zunächst die meist konzentrische Einengungdes Gesichtsfeldes [Vogt ${ }^{2}$ ], wenn auch, wie die Untersuchungen $0 \operatorname{ttos}^{3}$ ) erweisen, bei einer Arteriosklerose der Opticusarterie unregelmäßige Ausfälle des Gesichtsfeldes sich einstellen können. Bei der konzentrischen Einengung des Gesichtsfeldes handelt es sich wohl nur um ein Ermüdungs- oder, noch besser gesagt, um ein Verlangsamungssymptom. Denn wie die Sprache verlangsamt erscheint, erfolgt auch die Auffassung optischer Eindrücke entsprechend langsamer.

Wichtig sind ferner etwaige Begleiterscheinungen von seiten des Herzens (Angina pectoris) und Befunde an den peripherischen Arterien, welche auf Arteriensklerose hinweisen, sowie das Verhalten des Blutdruckes und der Nieren.

Allerdings muß ich betonelı, daß alle Erscheinungen, welche auf eine peripherische Arteriosklerose oder eine Arteriosklerose in anderen 0rganen hinweisen, durchaus noch keinen bindenden Schluß auf das Verhalten der Arterien des Zentralnervensystems zulassen.

Wir sehen also, dab eine ganze Reihe von nervösen Erscheinungen die Arteriosklerose des Zentralnervensystems begleiten kann. Es handelt sich dabei aber meist nicht um einfache Erscheinungen der Nervosität, sondern bei genauerem Zusehen finden wir immer eine ganze Reihe von Ausfalls- und Reizerscheinungen, welche nur auf eine Neuronerkrankung organischen Ursprungs zurückgeführt werden können. Einen Neurastheniker, einen endogen Nervösen(Degenerativen), welcher solche Erscheinungen in größerer Zahl bietet, gibt es nicht.

Die Differentialdiagnose ist deshalb gegenüber den reinen nervösen Zuständen nicht schwer, wenn man nur daran festhält, daß bei der Nervosität Zeichen einer organischen Erkrankung des Nervensystems nicht vorkommen.

Ich kann deshalb auch nicht anerkennen, dab, wie man

1).A. Cramer, Die Behandlung der Arteriosklerose. Münchener medizinische Wochenschrift 1907 und die Behandlung der arteriosklerotischen Atrophie des Großhirns. Deutsche medizinische Wochenschrif 1 1907, No. 47. - 2) Vog t, Gesichtsfeldeinengung bei Arteriosklerose. Allgemeine Zeitschrift für Psychiatrie, Bd. 59, S. 813 . 3) 0 t to, Sehnervenveränderungen bei Arteriosklerose. Berlin 1893, bei Springer. häufig liest, eine Neurasthenie oder ein anderer nervöser Zustand die Arteriosklerose des Zentralnervensystems einleitet. Untersuchen wir solche nervösen Zustände, welche der Arteriosklerose vorausgehen, genauer, so wird sich allerdings nicht, wenn wir uns auf eine einmalige Untersuchung beschränken, sondern nur wenn wir öfters untersuchen und genauer beobachten, bald das eine, bald das andere Symptom einstellen, das uns auf die organische Grundlage hinweist, bis allmählich die Diagnose immer deutlicher wird. Ich gehe auf diesen Punkt nicht etwa aus rein doktrinären Gründen ein, sondern deswegen, weil bei frühzeitiger Diagnose unsere Therapie in vielen Fällen durchaus nicht machtlos ist. Beruhigt man sich aber bei der Allgemeindiagnose Neurasthenie, läßt man die Sache gehen, dann kommt gewöhnlich erst die Diagnose Arteriosklerose, wenn es fast oder ganz zu spät ist, wie wir vielfach in unserer Sprechstunde sehen.

Es ist viel weniger gefährlich, einen einfachen Nervösen eine Zeitlang für einen Arteriosklerotiker zu halten, als einen Arteriosklerotiker für einen Nervösen. Dabei muß man sich auch darüber klar sein, daß auch gelegentlich ein endogen Nervöser, der zeitlebens nervös war, wenn er in das betreffende Alter kommt, eine Arteriosklerose des Zentralnervensystems bekommen kann. Geradeso wie eine Tabes, die langsam verläuft, sich nicht selten mit Arteriosklerose kompliziert.

Ich wende mich jetzt zum zweiten Teil meines Referates, zur Besprechung der psychischen Störungen bei der Ar teriosklerose des Zentralnervensystems. Namentlich durch die Untersuchungen Alzheimers, Binswangers, Webers und anderer ${ }^{1}$ ) ist pathologisch-anatomisch sichergestellt, dab die Arteriosklerose im Zentralnervensystem und speziell im Gehirn herdweise und diffus auftreten kann, daß beide Verbreitungsmethoden gleichzeitig vorhanden sein können und daß bei herdförmigem Auftreten der Krankheit die größeren und kleinen Herde und Herdehen mit ihren sekundären Degenerationen schließlich so zahlreich auftreten können, daß auch diese herdförmige Arteriosklerose das Gehirn in seiner Totalität ähnlich schädigt wie eine diffuse Erkrankung.

Die psychischen Störungen, welche wir beobachten, zerfallen in drei Gruppen:

1. Zunehmende Abnahme der geistigen Kräfte.

2. Leichte und schwere Depressionszustände.

3. Euphorische und Exaltationszustände, selten auch paranoische Symptomenkomplexe.

Was zunächst den einfachen Verfall der intellektuellen Fähigkeiten betrifft, so entwickelt sich derselbe meist langsam und schleichend, namentlich wenn wir die diffuse Form der Gehirnarteriosklerose vor uns haben. Häufig ist der Zustand durch das Vorherrschen schwerer Gedächtnisstörungen charakterisiert. Die Abnahme der Intelligenz erfolgt überhaupt in der Regel nicht gleichmäßig, meist bleiben vielmehr einzelne intellektuelle Reste und Inseln noch lange bestehen, ähnlich wie es Redepenning ${ }^{2}$ ) bei anderen Dementen geschildert hat. Gerade bei solchen Individuen, welche früher durch große geistige Ueberlegenheit und Schlagfertigkeit sich ausgezeichnet haben, sehen wir nicht selten, daß ganze Mechanismen der früheren Redetechnik und der früheren Kampfesweise (z. B. bei Rechtsanwälten) noch übrig bleiben und den Unerfahrenen täuschen, während der Erfahrenere bei genauerem Zusehen leicht feststellen kann, daß es sich nicht um neu gebildete Urteile und Schlüsse handelt, sondern lediglich um Reste von erhaltenen früher gebildeten Vorstellungskomplexen, welche mechanisch reproduziert werden. In dieses Gebiet gehören auch die schwachsinnigen Arteriosklerotiker, welche verhältnismäßig gut Skat spielen.

Das erste Zeichen der sich ausbildenden geistigen Schwäche ist häufig das Hervortreten ethischer Defekte und moralischer Perversitäten, welche früher bei dem $\mathrm{Pa}$ tienten nicht aufgefallen oder sicher nicht vorhanden waren. Wir sehen in solchen Fällen, daß Männer von bisher tadel-

1) B inswanger, Die Abgrenzung der progressiven Paralyse. Berliner klinische Wochenschrift 1894. A. C $\mathrm{r}$ a m e r, Pathologische Anatomie der Psychosen. Flatau und Jacobsohns Handbuch. - 2) Redepenning, Ziehens Monatschrift 1908, Bd. 23, Ergänzungsheft. 
losem Ruf, die sich nie das geringste haben zu Schulden kommen lassen, ganz im Gegensatz zu ihrem bisherigen ehrenwerten Verhalten Betrügereien und Urkundenfälschungen begehen, es mit der ehelichen Treue nicht mehr genau nehmen und ihre Familie nach jeder Richtung vernachlässigen. Kommt dazu noch die oben erwähnte Reizbarkeit in gesteigertem Maße, stellt sich gleichzeitig eine starke Urteilschwäche ein, dann kann das Schicksal der Familie und das Leben mit dem Vater zusammen geradezu „eine Hölle" darstellen. Dabei haben die Kranken oft noch die Fähigkeit, sich Fremden gegenüber zusammenzunehmen und namentlich den Laien mit den erhaltenen Intelligenzresten zu täuschen, sodaß die Entmündigung und namentlich die Ehescheidung die größten Schwierigkeiten machen. Ich habe in der vierten Auflage meiner "Gerichtlichen Psychiatrie" diese Verhältnisse eingehend erörtert und die Wege angegeben, welche uns das Gesetz zur Verfügung gestellt hat, um noch schlimmeres Unheil zu verhüten.

Zur Diagnose verhelfen uns in diesen Fällen nur eine genaue neurologische Untersuchung, exakte klinische Beobachtung und die moderne Intelligenzprüfung, wenn sie nicht $\mathrm{zu}$ schematisch angewandt wird. Es kommen hier die Methoden in Betracht, wie sie Rieger, Ziehen, Sommer und ich selbst angegeben haben und wie sie auch in der oben zitierten Arbeit von Redepenning geschildert worden sind.

Weniger Schwierigkeiten macht uns die Diagnose der Krankheit in diesen allerersten Stadien der psychischen Erkrankung, wenn der allmähliche Verlust des geistigen Kapitals sich von vornherein mit einer zunehmenden Stumpfheit und Indolenz auf intellektuellem und affektivem Gebiete verbindet. Es kommt hier höchstens die Differentialdiagnose der progressiven Paralyse gegenüber in Betracht, auf die ich erst später eingehen will. Manchmal verbindet sich mit diesen Zuständen auch eine auffällige Schlafsucht. Die Kranken schlafen, wenn man sie allein läbt, wenn die Anregung von außen fortfällt, sofort ein und machen manchmal auch in wachem Zustande einen somnolenten, benommenen, ja fast soporösen Eindruck. Die Fälle sind nicht selten, wo sich der gesamte Symptomenkomplex lediglich unter diesen Erscheinungen abspielt und allmählich zu einem vollständigen geistigen und körperlichen Verfall führt. Es ist das gewöhnlich auch das Ende der Krankheit, wenn sich häufende Herderscheinungen allmählich das Gehirn diffus schädigen.

Auffallendere psychische Störungen sind in den Fällen, wo die Krankheit von vornherein mit ausgeprägten Herderscheinungen kompliziert ist, selten. Auch möchte ich darauf hinweisen, dab zunächst ein oder sogar mehrere Insulte mit Herderscheinungen die intellektuelle Leistungsfähigkeit noch nicht in Mitleidenschaft zu ziehen brauchen. Wir dürfen deshalb, wenn in einem solchen Falle nach Jahren die Sektion erweist, daß das gesamte Gehirn durch zahllose kleinere und größere Herde diffus geschädigt ist, den Schlub ziehen, daß auch bereits nach den ersten Insulten eine derartige diffuse Schädigung mit entsprechender Beeinträchtigung der Intelligenz bestanden habe. Soll das angenommen werden, dann müssen auch die entsprechenden eben geschilderten klinischen Erscheinungen vorliegen. Diese Tatsache ist bei der Frage der Geschäfts- und Testierfähigkeit in solchen Fällen wichtig.

Die häufigste psychische Anomalie, die man auf dem Boden der Arteriosklerose beobachtet, sind die Depressionszustände. Sie leiten sich nicht selten durch ein hypochondrisches Stadium ein. Die Kranken leiden, wie das auch Weber hervorhebt, schwer unter der Furcht, unheilbar krank zu sein, sie gehen von einem Arzt zum andern und lassen sich untersuchen, sie machen sich namentlich Sorgen wegen ihres Stuhlganges und der Urinentleerung, sie denken an eine frühere Infektion und sprechen von Gehirnerweichung, andere wieder halten sich für übelriechend, für giftig für die Umgebung; ein sehr feingebildeter Mann meiner Praxis beklagte sich bei mir im Beginn seines Depressionszustandes, er esse so unappetitlich und könne deshalb nicht mehr unter Menschen gehen. Ueberhaupt leitet häufig eine ausgeprägte Menschenscheu, eine Neigung sich zurückzuziehen in Verbindung mit einem starken Insuffizienzgefühl den eigentlichen Depressionszustand ein. Gewöhnlich wird der Depressionszustand noch verstärkt, wenn die Patienten von Unglücksfällen betroffen werden. Aber auch ohne solche äuberen Ereignisse nimmt die Verstimmung rasch zu und kann sich bis zur Verzweiflung steigern. Selbstmordversuche sind in diesem Stadium nicht selten und führen die Kranken in die geschlossenen Anstalten, während sie bis dahin in offenen Kuranstalten und Sanatorien oder in Kurpfuscherinstituten verpflegt worden sind. Oft, aber durchaus nicht immer, äußern die Kranken dabei starke Angst und machen sich die stärksten Selbstvorwürfe oder äußern hypochondrische Ideen, ähnlich wie sie Paralytiker im Depressionsstadium vorbringen.

Es liegt nahe, hier die Differentialdiagnose der Melancholie gegenüber zu berühren. Denn sicher sind viele dieser Fälle früher unter dem Namen Melancholie gegangen. Bei genauerer Betrachtung fällt uns aber auf, daß bei diesen arteriosklerotischen Depressionen die Kranken im allgemeinen etwas stumpfer erscheinen, daß ihre Aeußerungen noch eintöniger als bei der echten Melancholie sind, dab gelegentlich Zustände von Stumpfheit und Benommenheit uns auffallen, und vor allen Dingen, dalb bei genauer neurologischer Prüfung stets einzelne Zeichen einer organischen Gehirnerkrankung mehr oder weniger deutlich ausgeprägt sich finden. Gerade diese Mischung mit, wenn auch nur leicht angedeuteten Symptomen einerorganischen Erkrankung des Zentralnervensystems gibt dem Depressionszustand ein so eigentümliches Gepräge, daß der Sachkundige, der diese Fälle kennt, sofort aufmerksam wird und nach diesem Zeichen fahndet, um zu einer klaren Diagnose zu kommen.

Die Dauer dieser Depressionszustände ist sehr verschieden. Es gibt eine ganze Reihe Fälle, welche, namentlich wenn rechtzeitig therapeutisch eingegriffen wird, nach einigen Wochen und Monaten sich wieder so weit erholen, daß sie des Aufenthaltes in einer geschlossenen Anstalt nicht mehr bedürfen. Man mul sich aber hüten, diese Rekonvaleszenten bei ungünstigen äußeren Verhältnissen bald wieder in ihren Beruf oder in ihre Familie zurückzuschicken. Denn genesen sind die Kranken nicht, es ist lediglich der Depressionszustand beseitigt, die Arteriosklerose ist geblieben. Sie bedürfen noch längerer Behandlung bei möglichster Ruhigstellung des Gehirns. Sind die äußeren Verhältnisse ungünstig, oder stürzt sich der Patient trotz allen Abratens wieder in das Geschäft, dann kommen bald wieder Rezidive oder auch Insulterscheinungen. Erlauben dagegen die Verhältnisse dem Kranken ein ruhiges Leben nach genau ärztlich angeordnetem und überwachtem Regime, dann kann die Krankheit für Jahre zum Stillstand kommen und dem Patienten ein Otium cum dignitate gestatten, das ihn seiner Familie erhält, ihm aber alle energischeren Genüsse des Lebens untersagt (Wein- und Tafelfreuden, sexueller Verkehr und sportliche Leistungen). Gerade in den Fällen, wo es den Angehörigen nicht rasch genug geht, wo der Arzt nicht genug erreicht und deshalb der Kurpfuscher zu Rate gezogen wird, sehen wir gewöhnlich einen rapiden ungünstigen Verlauf, sei es, dab der Patient zuviel mit kaltem Wasser und Gymnastik und anderen schädlichen Prozeduren traktiert wird, sei es, daß er auf der Fahrt nach dem Hochgebirge oder der See an einem heißen Tage der Hitze im Eisenbahncoupee zum 0pfer fällt.

Forschen wir nach der Ursache, weshalb wir gerade Depressionszustände bei der Arteriosklerose so häufig sehen, so liegt es nahe, hier an die Beziehungen der Gefäbe zu den Affekten zu denken. Denn gerade die Gefäße, welche bei den Affekten besonders in Betracht kommen, die Arterien sind es, welche erkrankt sind. ${ }^{1}$ ) Ich möchte hier auf eine Publikation von Rosenfeld ${ }^{2}$ ) hinweisen, der andeutet, daß in einzelnen seiner Fälle von vasomotorischer Neurose auch eine Arteriosklerose in Betracht kommen mub, und betone, dab Weber mit Recht hervorhebt, dal in vielen Fällen von Arteriosklerose eine ausgesprochene Gefäßbelastung besteht.

In der dritten Gruppe von psychischen Störungen, welche ich abgegrenzt habe, finden wir euphorische und Exaltationszustände sowie paranoische Symptomenkomplexe. Bei dieser Gruppe finden wir häufig, wie das auch Weber

1) A. Cramer, Zur Theorie der Affekte. Göttingen, Kösier, 1908. - 2) Rosenfeld, Vasomotorische Neurose. Zentralblatt für Nervenheilkunde 1909, s 137. 
kürzlich wieder betont hat, daß die individuelle psychische Veranlagung ins Krankhafte verzerrt und gesteigert zum Ausdruck kommt. Ich habe eine ganze Reihe von Fällen gesehen, wo auf degenerativem Boden von jeher eine.sehr leichte Lebensauffassung bestanden hat und namentlich das Jagen nach dem Genuß zur obersten Richtschnur alles Handels gemacht worden war. Hier mußte, als infolge der Arteriosklerose das Können nachlieb, das Reden und Renommieren namentlich über sexuelle Abenteuer die Wirklichkeit ersetzen. Die Kranken wurden mit dem Auftreten einer gewissen Euphorie immer lasziver in ihren Reden, nahmen auf Frau und erwachsene Töchter nicht die geringste Rücksicht, trieben sich mit den Damen vom horizontalen Metier in breitester Oeffentlichkeit herum und ruinierten sich ähnlich wie ein beginnender Paralytiker Stellung und Ansehen vollständig. Auch später während der Anstaltsbehandlung trat die Parallele mit der Paralyse in zeitweise auftretenden Größenideen deutlich hervor. Charakteristisch ist ferner diesen Fällen eine zeitweise auftretende Sucht zum Renommieren. Gerade diese Kranken sind häufig Menschen, denen im früheren Leben der Schein immer über das Sein ging. Weber hat charakteristische, entsprechende Fälle aus meiner Klinik mitgeteilt. Charakteristisch ist auch für die Kranken dieser Gruppe, daß der ProzeB in Remis sionen verläuft, daß ruhigere Intervalle kommen, daß die Angehörigen sich täuschen lassen und eine Genesung annehmen und daß draußen sehr bald wieder eine neue Exazerbation einsetzt.

Die paranoischen Symptomenkomplexe kennzeichnen sich meistens in einem immer mehr sich steigernden Mißtrauen gegen die nächste Umgebung und speziell gegen die Angehörigen. Allmählich wird aus dem gesteigerten Mißtrauen die Wahnidee der Verfolgung, denen entsprechend die Kranken reagieren. In selteneren Fällen werden diese Verfolgungsideen auch durch entsprechende Sinnestäuschungen, namentlich durch Gehörstäuschungen unterstützt.

In zwei Fällen sah ich bei Patienten, welche bisher psychische Störungen noch nicht hatten erkennen lassen, plötzlich Gehörstäuschungen auftreten, welche die Patienten lebhaft beunruhigten, sie ganze Unterhaltungen, ähnlich wie Alkoholiker (Kraepelin) über sich hören ließen und zu energischen $A b w e h r m a ß r$ regeln veranlaßten. In einem Falle Anzeige an die Staatsanwaltschaft gegen den vermeintlichen Urheber, im anderen Falle Forderung des vermeintlichen Beleidigers auf Pistolen. Diese halluzinatorischen Attacken traten anfangs nur selten, oft nur für ein bis zwei Stunden in einer Woche auf, um dann allmählich häufiger zu kommen. Länger dauernd bestanden sie aber nie. Es kamen immer wieder Intervalle, in denen die Kranken völlig objektiv über diese Erscheinungen urteilten. Beide Fälle erlitten später, das heißt nach einigen Monaten, eine Reihe von Insulten, welche rasch ihre Intelligenz stark reduzierten.

Ueberhaupt ist der weitere Verlauf vieler Fälle von psychischer Störung von Arteriosklerose dadurch charakterisiert, daß schließlich namentlich apoplektische Insulte, welche in größerer Zahl auftreten, rasch Verblödung herbeiführen.

An Stelle der Insulte können auch Reizerscheinungen auftreten, welche manchmal ähnlich wie bei dem paralytischen Anfall den Jacksonschen Typus zeigen. Ferner können dauernde aphasische Zustände und Hemianopsien das Krankheitsbild komplizieren. Auch interkurrente Zustände schwerer Benommenheit, welche mit Fieberbewegungen einhergehen, können auftreten.

Ich habe bisher wiederholt auf Aehnlichkeiten und Parallelen mit der progressiven Paralyse hingewiesen. Es wird zum Schluß noch meine Aufgabe sein zu untersuchen, ob es uns heute möglich ist, die progressive Paralyse von den psychischen Störungen bei arteriosklerotischer Atrophie zu trennen.

Wenn es auch Fälle gibt, bei denen man lange Zeit zwischen den beiden Diagnosen schwankt, so glaube ich doch, daß man im allgemeinen diese Frage bejahen kann. Was zunächst die Krankheitsdauer betrifft, so ist namentlich das nervöse Prodromalstadium meist erheblich länger als das der progressiven Paralyse, auch sind, so lange diese nervösen Beschwerden ohne ausgesprochene psychische Störungen bestehen, bei der Paralyse immer einige der körperlichen Kar- dinalsymptome mehr oder weniger deutlich markiert vorhanden, ganz abgesehen davon, daß sich auch eine ausgesprochene Charakterveränderung in der Regel viel deutlicher, früher und ausgeprägter bei der progressiven Paralyse markiert als bei der Arteriosklerose. Die Krankheit trifft bei der Paralyse viel eher den Kern der ganzen Persönlichkeit. (Weber.) Finden sich bei der Arteriosklerose sehr frühzeitig $\mathrm{Pu}$ pillunveränderungen, so ist im Gegensatz zur progressiven Paralyse meist charakteristisch, daß fast gleichzeitig die Reaktion auf Lichteinfall und Akkommodation nachläßt (Weber) und daß verhältnismäßig erst spät eine totale Starre eintritt. Im Gegensatz zur Paralyse setzt die Krankheit meist in einem höheren Alter ein, auf einem Boden, bei dem sehr häufig eine degenerative Veranlagung und eine starke Gefäßbelastung (Weber) besteht. Auch verläuft die Arteriosklerose des Zentralnervensystems viel mehr in Schwankungen als die progressive Paralyse, und wenn auch bei beiden Remissionen vorkommen können, so sind sie doch bei der Paralyse häufiger.

Die Wassermannsche Serumreaktion kann aus dem Blute auch bei der arteriosklerotischen Atrophie positiv sein, wenn der Träger der Atrophie früher eine Lues durcligemacht hat. Sie ist aber aus der Spinalflüssigkeit ebenso wie die Nonnesche Reaktion so gut wie nur bei der Paralyse und Tabes und in einigen Fällen von Hirnlues positiv. Wir haben also in der Wassermannschen Serumreaktion der Spinalflüssigkeit ein gutes differentialdiagnostisches Moment, um die Arteriosklerose des Zentralnervensystems von der progressiven Paralyse zu trennen. ${ }^{1)}$

In Betracht kann nur noch die Lues cerebrospinalis kommen. Aber auch sie ist meist ohne Schwierigkeiten durch die Reaktion auf die spezifische Therapie abzutrennen, ganz abgesehen davon, daß in ausgeprägten Fällen die Diagnose an und für sich keine Schwierigkeiten macht.

Wir haben also gesehen, daß die Arteriosklerose des Zentralnervensystems in wohl umschriebenen Krankheitsbildern sowohl auf nervösem als psychischem Gebiete klinisch zum Ausdruck kommt. Die Kenntnis dieser Krankheitsbilder ist nicht nur von klinisch-wissenschaftlichem Interesse, sondern auch praktisch von großer Bedeutung, weil bei möglichst frühzeitiger Diagnose durch eine entsprechende Therapie die Krankheit in nicht wenigen Fällen zum Stillstand gebracht werden kann und sozialer und materieller Schaden für den Patienten und die Angehörigen vermieden wird. 